\title{
APPROXIMATION THEOREMS FOR ZERO-SUM NONSTATIONARY STOCHASTIC GAMES
}

\author{
ANDRZEJ S. NOWAK
}

\begin{abstract}
This paper deals with zero-sum nonstationary stochastic games with countable state and action spaces which include both Shapley's stochastic games $[\mathbf{1 1}]$ and infinite games with imperfect information studied by Orkin in [7]. It is shown that any nonstationary stochastic game with a bounded below lower semicontinuous payoff defined on the space of all histories has a value function and the minimizer has an optimal strategy. Moreover, two approximation theorems extending the main results of Orkin from $[\mathbf{7}]$ are established. Finally, counterexamples answering in the negative some open questions raised by Orkin $\{\mathbf{7}]$ and Sengupta $[\mathbf{1 0}]$ are given.
\end{abstract}

1. Introduction. Before we describe our game model, let us accept some conventions. Let $N$ denote the set of positive integers, and $R$ the set of real numbers. If $f, g: S \rightarrow R$ are functions on a nonempty set $S$, then $f \leq g$ means that $f(s) \leq$ $g(s)$ for each $s \in S$. If $S$ is a countable set, then by $P(S)$ we denote the set of all probability measures on the $\sigma$-algebra of all subsets of $S$. Finally, throughout the sequel we shall - tacitly - assume that any countable set is endowed with the discrete topology, and the countable product of such sets is endowed with the usual product topology.

A nonstationary two-person zero-sum stochastic game $G$ which we consider is defined by a two-person stochastic control system $\left\{S_{n}, X_{n}, Y_{n}, q_{n}, u ; n \in N\right\}$ where:

(i) $S_{n}$ is the state space of the system at stage $n \in N$. $S_{n}$ is assumed to be a nonempty countable set.

(ii) $X_{n}$ and $Y_{n}$ are the action spaces for players I and II, respectively, at stage $n \in N$. It is assumed that $X_{n}\left(Y_{n}\right)$ is a nonempty countable (finite) set.

Let $H_{1}=S_{1}, H_{n}=S_{1} \times X_{1} \times Y_{1} \times \cdots \times S_{n}$, and $H_{\infty}=S_{1} \times X_{1} \times Y_{1} \times S_{2} \times$ $X_{2} \times Y_{2} \times \cdots$. Then $H_{n}$ is the set of histories up to stage $n \in N$, and $H_{\infty}$ is the set of all histories of the game.

(iii) $\left\{q_{n}\right\}$ is the law of motion of the system, i.e., $q_{n}: H_{n} \times X_{n} \times Y_{n} \rightarrow P\left(S_{n+1}\right)$ is a transition probability such that $q_{n}\left(h_{n}, x_{n}, y_{n}, \cdot\right)$ is the condition distribution of the stage of the system at stage $n+1$ given the history $h_{n} \in H_{n}$ and the actions $x_{n} \in X_{n}$ and $y_{n} \in Y_{n}$ chosen by the players at stage $n$.

(iv) $u: H_{\infty} \rightarrow R$ is the payoff function. It is assumed that $u$ is a bounded below lower semicontinuous (l.s.c. in short) function on $H_{\infty}$.

The game is played as follows. The players I and II observe the initial state $s_{1} \in$ $S_{1}$ of the system and choose simultaneously actions $x_{1} \in X_{1}$ and $y_{1} \in Y_{1}$, respectively. Then the result $\left(x_{1}, y_{1}\right)$ is announced to both of them and the system moves

Received by the editors November 20, 1983.

1980 Mathematics Subject Classification. Primary 90D15, 90D13; Secondary 90D05, 93E05.

Key words and phrases. Zero-sum discrete-time nonstationary stochastic game, infinite game, imperfect information. 
to a new state $s_{2} \in S_{2}$ according to the probability distribution $q_{1}\left(s_{1}, x_{1}, y_{1}, \cdot\right)$, upon which I chooses $x_{2} \in X_{2}$ while II chooses $y_{2} \in Y_{2}$, etc. The result of this infinite sequence of moves is a point $h=\left(s_{1}, x_{1}, y_{1}, s_{2}, x_{2}, y_{2}, \ldots\right) \in H_{\infty}$ and II pays I the amount $u(h)$.

For each $n \in N$, let $\Pi_{n}\left(\Gamma_{n}\right)$ be the set of all functions (transition probabilities) from $H_{n}$ to $P\left(X_{n}\right)\left(P\left(Y_{n}\right)\right)$.

A (behavioural) strategy for player I (II) is a sequence $\pi=\left\{f_{n}\right\}\left(\gamma=\left\{g_{n}\right\}\right)$, where $f_{n} \in \Pi_{n}\left(g_{n} \in \Gamma_{n}\right)$ for each $n \in N$. We denote by $\Pi(\Gamma)$ the set of all strategies for player I (II).

According to the theorem of Ionescu-Tulcea (see [3, p. 149 or 5, Proposition V 1.1]), for each pair $\pi=\left\{f_{n}\right\}, \gamma=\left\{g_{n}\right\}$ of strategies there exists a unique conditional probability $P_{\pi \gamma}\left(s_{1}, \cdot\right)$ on $K=X_{1} \times Y_{1} \times S_{2} \times X_{2} \times Y_{2} \times S_{3} \times X_{3} \times Y_{3} \times \cdots$ given the initial state $s_{1}$ such that for each cylindrical set $C_{n}=\left\{x_{1}\right\} \times\left\{y_{1}\right\} \times \cdots \times\left\{s_{n}\right\} \times$ $\left\{x_{n}\right\} \times\left\{y_{n}\right\} \times\left\{s_{n+1}\right\} \times X_{n+1} \times Y_{n+1} \times \cdots \subset K$ we have

$$
P_{\pi \gamma}\left(s_{1}, C_{n}\right)=\prod_{k=1}^{n} f_{k}\left(h_{k},\left\{x_{k}\right\}\right) g_{k}\left(h_{k},\left\{y_{k}\right\}\right) q_{k}\left(h_{k}, x_{k}, y_{k},\left\{s_{k+1}\right\}\right),
$$

where $h_{1}=s_{1}, h_{k}=\left(s_{1}, x_{1}, y_{1}, \ldots, s_{k}\right), 2 \leq k \leq n$.

Thus, each pair $\pi, \gamma$ defines an expected payoff to player I in the game $G$ at an initial state $s_{1} \in S_{1}$ to be

$$
E(u, \pi, \gamma)\left(s_{1}\right)=\int u\left(s_{1}, h\right) P_{\pi \gamma}\left(s_{1}, d h\right)
$$

From (iv), it follows that $E(u, \pi, \gamma)$ is a bounded below extended real-valued function of the initial state.

Define, for each $s_{1} \in S_{1}$,

$$
L(G)\left(s_{1}\right)=\sup _{\pi \in \Pi} \inf _{\gamma \in \Gamma} E(u, \pi, \gamma)\left(s_{1}\right)
$$

and

$$
U(G)\left(s_{1}\right)=\inf _{\gamma \in \Gamma} \sup _{\pi \in \Pi} E(u, \pi, \gamma)\left(s_{1}\right) .
$$

Then $L(G)(U(G))$ is called the lower (upper) value function of the game $G$. It is always true that $L(G) \leq U(G)$. If $L(G)=U(G)$, this common function is called the value function of the game $G$ and will be denoted by $\operatorname{Val}(G)$.

Throughout the sequel we shall assume that there exists a strategy $\gamma \in \Gamma$ such that

$$
\sup _{\pi \in \Pi} E(u, \pi, \gamma)\left(s_{1}\right)<\infty \quad \text { for each } s_{1} \in S_{1} .
$$

Let $\varepsilon \geq 0$ be given and suppose the value function $\operatorname{Val}(G)$ exists.

A strategy $\pi^{*} \in \Pi$ is called $\varepsilon$-optimal for player I if

$$
\operatorname{Val}(G) \leq \inf _{\gamma \in \Gamma} E\left(u, \pi^{*}, \gamma\right)+\varepsilon
$$

A strategy $\gamma^{*} \in \Gamma$ is called $\varepsilon$-optimal for player II if

$$
\operatorname{Val}(G) \geq \sup _{\pi \in \Pi} E\left(u, \pi, \gamma^{*}\right)-\varepsilon
$$


REMARK 1.1. Stochastic games were introduced by Shapley [11]. He considered a special case of the game described above in which $S_{n}=S, X_{n}=X$, and $Y_{n}=Y$ for each $n \in N$ and some finite sets $S, X$, and $Y$. The law of motion $\left\{q_{n}\right\}$ in Shapley's game is stationary, that is, $q_{n}\left(h_{n}, x_{n}, y_{n}, \cdot\right)=q\left(s_{n}, x_{n}, y_{n}, \cdot\right)$ for each $h_{n}=\left(s_{1}, x_{1}, y_{1}, \ldots, s_{n}\right) \in H_{n}, x_{n} \in X_{n}, y_{n} \in Y_{n}$ and some $q: S \times X \times Y \rightarrow P(S)$. Moreover, the payoff is accumulated over stages with a discount factor $\beta(0 \leq \beta<$ 1), so that for each history $h=\left(s_{1}, x_{1}, y_{1}, \ldots\right) \in H_{\infty}$,

$$
u(h)=\sum_{n=1}^{\infty} \beta^{n-1} r\left(s_{n}, x_{n}, y_{n}\right)
$$

where $r$ is a (payoff per stage) function on $S \times X \times Y$. (Of course, (1) is continuous on $H_{\infty}$.) Such a game is called a discounted Markov (or stationary stochastic) game. (For further results concerning Markov games we refer to $[\mathbf{1}, \mathbf{4}, \mathbf{6}, \mathbf{8}]$ and the references therein.)

REMARK 1.2. Let $S_{n}=\{n\}$ and let $X_{n}$ be finite for each $n \in N$. Then our game reduces itself to an infinite game with imperfect information studied by Orkin in [7]. The goal of this paper is to extend Orkin's approximation theorems for infinite games from [7] to nonstationary stochastic games defined above.

REMARK 1.3. The game introduced here is inspired by a nonstationary stochastic control system in the sense of Hinderer [3, Chapter I]. Some different nonstationary stochastic games were studied by Sengupta [10] and Schäl [9]. In the model of Sengupta [10] the state space is an independent of time compact metric space, the action spaces are finite, the payoff is l.s.c. on the set of all histories, but the law of motion is stationary. Schäl has considered in [9] a game model with Borel state and action spaces in which player I, similarly, player II, may base his decision at any stage $n$ on the whole history $\left(s_{1}, s_{2}, \ldots, s_{n}\right)$ of the system and on his own previous actions only. In other words, each of the players cannot take into account at any stage the previous choices of his opponent. Such a restriction concerning the players does not take place in our model.

2. Finite horizon games. Let $m \in N$ be fixed and let $u_{m}$ be a bounded below real-valued function on $Z_{m}=H_{m} \times X_{m} \times Y_{m}$. Then $u_{m}$ may be recognized as a continuous function on $H_{\infty}$ and it may be considered as a payoff function of some nonstationary stochastic game in the sense of $\S 1$. Such a game is called a finite horizon game because the payoff is decided here in the first $m$ moves.

We have the following fact.

PROPOSITION 2.1. Every finite horizon nonstationary stochastic game satisfying (i)-(v) has a value function. Moreover, for each $\varepsilon>0$, player I has an $\varepsilon$-optimal strategy and player II has an optimal strategy.

Before we prove Proposition 2.1, let us introduce some auxiliary operators. Let $w: Z_{n} \rightarrow R$ be bounded below, $f_{n} \in \Pi_{n}$, and $g_{n} \in \Gamma_{n}, n \in N$. Then we put

$$
\begin{aligned}
\left(A_{f_{n} g_{n}} w\right)\left(h_{n}\right)=\sum_{x_{n} \in X_{n}} \sum_{y_{n} \in Y_{n}} w\left(h_{n}, x_{n}, y_{n}\right) f_{n}\left(h_{n},\left\{x_{n}\right\}\right) g_{n}\left(h_{n},\left\{y_{n}\right\}\right), & \\
& h_{n} \in H_{n}
\end{aligned}
$$


Let $w: H_{n+1} \rightarrow R$ be bounded below. Then we put

$$
\begin{array}{r}
\left(Q_{n} w\right)\left(h_{n}, x_{n}, y_{n}\right)=\sum_{s_{n+1} \in S_{n+1}} w\left(h_{n}, x_{n}, y_{n}, s_{n+1}\right) q_{n}\left(h_{n}, x_{n}, y_{n},\left\{s_{n+1}\right\}\right), \\
\left(h_{n}, x_{n}, y_{n}\right) \in H_{n} \times X_{n} \times Y_{n} .
\end{array}
$$

Note that $A_{f_{n} g_{n}}$ is isotone for each $f_{n}, g_{n}$, i.e., $A_{f_{n} g_{n}} w \leq A_{f_{n} g_{n}} v$ if $w \leq v$, and $A_{f_{n} g_{n}}(w+c)=A_{f_{n} g_{n}} w+c$ for any constant $c$. Similar facts hold for $Q_{n}, n \in N$.

Let $\pi=\left\{f_{n}\right\} \in \Pi$ and $\gamma=\left\{g_{n}\right\} \in \Gamma$. It can easily be verified that for any finite horizon game with the payoff function $u_{m}$ we have

$$
E\left(u_{m}, \pi, \gamma\right)=A_{f_{1} g_{1}} Q_{1} A_{f_{2} g_{2}} Q_{2} \cdots A_{f_{m-1} g_{m-1}} Q_{m-1} A_{f_{m} g_{m}} u_{m}
$$

Now we are ready for the

PROOF OF PROPOSITION 2.1. The proof proceeds by induction and is similar to that of Lemma 3.5 from [1]. Since the payoff in an $m$-stage game is decided in the first $m$ moves, so we can restrict our attention to the first $m$ terms of strategies only. For the one-stage game the result follows directly from Fan's minimax theorem $[\mathbf{2}$, Theorem 2]. Fix $m \geq 2$ and suppose the result holds for every $(m-1)$-stage game. Let $\varepsilon>0$ be given and let $u_{m}$ be a payoff function of an $m$-stage game $G_{m}$. From Fan's minimax theorem [2] we infer that

$$
v_{m}:=\sup _{f_{m} \in \Pi_{n}} \inf _{g_{m} \in \Gamma_{m}} A_{f_{m} g_{m}} u_{m}=\inf _{g_{m} \in \Gamma_{m}} \sup _{f_{m} \in \Pi_{m}} A_{f_{m} g_{m}} u_{m}
$$

and, moreover, we can find $f_{m}^{*} \in \Pi_{m}$ and $g_{m}^{*} \in \Gamma_{m}$ such that, for every $f_{m} \in \Pi_{m}$ and $g_{m} \in \Gamma_{m}$,

$$
A_{f_{m} g_{m}^{*}} u_{m} \leq v_{m} \quad \text { and } \quad v_{m} \leq A_{f_{m}^{*} g_{m}} u_{m}+\varepsilon / 2 .
$$

Now, let $G_{m-1}$ be the $(m-1)$-stage game where the payoff function is given by $u_{m-1}=Q_{m-1} v_{m}$. From our induction hypothesis, it follows that $G_{m-1}$ has a value function and there exist strategies $\pi_{m-1}^{*}=\left(f_{1}^{*}, f_{2}^{*}, \ldots, f_{m-1}^{*}\right)$ and $\gamma_{m-1}^{*}=$ $\left(g_{1}^{*}, g_{2}^{*}, \ldots, g_{m-1}^{*}\right)$ for I and II such that

$$
E\left(u_{m-1}, \pi, \gamma_{m-1}^{*}\right) \leq \operatorname{Val}\left(G_{m-1}\right) \leq E\left(u_{m-1}, \pi_{m-1}^{*}, \gamma\right)+\varepsilon / 2,
$$

for every $\pi \in \Pi$ and $\gamma \in \Gamma$.

Define $\pi_{m}^{*}=\left(f_{1}^{*}, f_{2}^{*}, \ldots, f_{m-1}^{*}, f_{m}^{*}\right)$ and $\gamma_{m}^{*}=\left(g_{1}^{*}, g_{2}^{*}, \ldots, g_{m-1}^{*}, g_{m}^{*}\right)$. Using (4), (5) and the elementary properties of the operators (2) and (3), one can check that

$$
E\left(u_{m}, \pi, \gamma_{m}^{*}\right) \leq E\left(u_{m-1}, \pi, \gamma_{m-1}^{*}\right) \quad \text { and } \quad E\left(u_{m-1}, \pi_{m-1}^{*}, \gamma\right) \leq E\left(u_{m}, \pi_{m}^{*}, \gamma\right)+\varepsilon / 2
$$

for all $\pi \in \Pi$ and $\gamma \in \Gamma$. Hence and from (6) we infer

$$
U\left(G_{m}\right) \leq \sup _{\pi \in \Pi} E\left(u_{m}, \pi, \gamma_{m}^{*}\right) \leq \inf _{\gamma \in \Gamma} E\left(u_{m}, \pi_{m}^{*}, \gamma\right)+\varepsilon \leq L\left(G_{m}\right)+\varepsilon .
$$

This implies that $G_{m}$ has a value function, $\gamma_{m}^{*}$ is an optimal strategy for player II and $\pi_{m}^{*}$ is an $\varepsilon$-optimal strategy for player I, which terminates the proof.

REMARK 2.1. Let $\gamma=\left\{g_{n}\right\} \in \Gamma$ be arbitrary and let $\gamma_{m}^{*}=\left\{g_{n}^{*}\right\}, n \leq m$, be an optimal strategy for player II in the $m$-stage game. Then $\gamma^{*} \in \Gamma$ defined by $\gamma^{*}=\left(g_{1}^{*}, g_{2}^{*}, \ldots, g_{m}^{*}, g_{m+1}, g_{m+2}, \ldots\right)$ is an optimal strategy for him too. 
3. Compactness of the set of strategies for player II. Let us assume that each set $\Gamma_{n}=\left\{g_{n}: H_{n} \rightarrow P\left(Y_{n}\right)\right\}$ is endowed with the topology of pointwise convergence. Since each set $Y_{n}$ is assumed to be finite, so each $P\left(Y_{n}\right)$ is a compact metric space. Thus, from Tychonoff's theorem and the countability of sets $H_{n}$, $n \in N$, we infer that each $\Gamma_{n}$ is a compact metrizable topological space. Now, let us endow $\Gamma=\Gamma_{1} \times \Gamma_{2} \times \cdots$ with the product topology. Then $\Gamma$ is a compact metrizable topological space too.

We shall need the following fact.

LEMMA 3.1. Consider a finite horizon game with a payoff $u_{m}$ defined on $Z_{m}=$ $H_{m} \times X_{m} \times Y_{m}$. Then, for each $s_{1} \in S_{1}$ and $\pi \in \Pi$, the function $E\left(u_{m}, \pi, \cdot\right)\left(s_{1}\right)$ is lower semicontinuous on $\Gamma$.

PROOF. First note that without loss of generality we may assume that $u_{m}$ is nonnegative. Then observe that under (i) and (ii) the set $Z_{m}$ may be represented as a denumerable sequence $\left\{z_{n}\right\}$. Let $w_{n}: Z_{m} \rightarrow R$ be defined as follows: $w_{n}(z)=u_{m}(z)$ if $z=z_{n}$ and $w_{n}(z)=0$ otherwise. Let $v_{n}=w_{1}+w_{2}+\cdots+w_{n}$, $n \in N$. Clearly, $v_{n} \nearrow u_{m}$ as $n \rightarrow \infty$. By the monotone convergence theorem, $E\left(v_{n}, \pi, \cdot\right)\left(s_{1}\right) \nearrow E\left(u_{m}, \pi, \cdot\right)\left(s_{1}\right)$ as $n \rightarrow \infty\left(s_{1} \in S_{1}, \pi \in \Pi\right)$. It is quite obvious that each function $E\left(v_{n}, \pi, \cdot\right)\left(s_{1}\right)$ is continuous on $\Gamma$. Thus, $E\left(u_{m}, \pi, \cdot\right)\left(s_{1}\right)$ as a limit of nondecreasing sequences of continuous functions on $\Gamma$ is l.s.c. on $\Gamma$, and this completes the proof.

Let $H^{n}=S_{n+1} \times X_{n+1} \times Y_{n+1} \times S_{n+2} \times X_{n+2} \times Y_{n+2} \times \cdots, n \in N$. Define $u_{m}: Z_{m} \rightarrow R$ by

$$
u_{m}\left(h_{m}, x_{m}, y_{m}\right)=\inf _{h \in H^{n}} u\left(h_{m}, x_{m}, y_{m}, h\right), \quad\left(h_{m}, x_{m}, y_{m}\right) \in Z_{m},
$$

where $u$ is the payoff function of the game $G$ from $\S 1$.

From assumption (iv) we can conclude the following fact.

LEMMA 3.2. If $m \rightarrow \infty$, then $u_{m} \nearrow u$ on $H_{\infty}$.

From Lemmas 3.1 and 3.2 and the monotone convergence theorem we infer the following important

COROLlaRY 3.1. For each $s_{1} \in S_{1}$ and $\pi \in \Pi$, the function $E(u, \pi, \cdot)\left(s_{1}\right)$ is lower semicontinuous on $\Gamma$.

4. Approximation theorems for infinite horizon games. This final section contains the main results of this paper. Here is one of them.

THEOREM 4.1. The game $G$ satisfying $(\mathrm{i})-(\mathrm{v})$ has a value function $\operatorname{Val}(G)$ and $\operatorname{Val}(G)=\lim _{n} \operatorname{Val}\left(G_{n}\right)$, where $G_{n}, n \in N$, are the finite horizon games with payoffs $u_{n}$ defined according to (7). Moreover, for any $\varepsilon>0$ player I has an $\varepsilon$-optimal strategy and player II has an optimal strategy.

Proof. Note that $\operatorname{Val}\left(G_{n}\right) \leq \operatorname{Val}\left(G_{n+1}\right) \leq L(G)$ for each $n \in N$. Hence $\lim _{n} \operatorname{Val}\left(G_{n}\right) \leq L(G)$. By Proposition 2.1 and Remark 2.1, for each $n \in N$, player II has an optimal strategy $\gamma_{n} \in \Gamma$ in $G_{n}$. Since $\Gamma$ is a compact metrizable space, so $\left\{\gamma_{n}\right\}$ contains a subsequence $\left\{\gamma_{n_{k}}\right\}$ converging to some $\gamma^{*} \in \Gamma$. Fix $m \in N$. Then for each $n_{k} \geq m$ we have

$$
E\left(u_{m}, \pi, \gamma_{n_{k}}\right) \leq \operatorname{Val}\left(G_{n_{k}}\right) \leq \lim _{n} \operatorname{Val}\left(G_{n}\right) \leq L(G)
$$


This and Corollary 3.1 imply that

$$
E\left(u_{m}, \pi, \gamma^{*}\right) \leq \liminf _{k} E\left(u_{m}, \pi, \gamma_{n_{k}}\right) \leq L(G), \quad \pi \in \Pi, m \in N .
$$

By Lemma 3.2 and the monotone convergence theorem

$$
E\left(u, \pi, \gamma^{*}\right)=\lim _{m} E\left(u_{m}, \pi, \gamma^{*}\right) \leq L(G), \quad \pi \in \Pi .
$$

Hence

$$
U(G) \leq \sup _{\pi \in \Pi} E\left(u, \pi, \gamma^{*}\right) \leq L(G) .
$$

Thus, the game $G$ has a value function and $\gamma^{*}$ is an optimal strategy for player II.

Now, let $\varepsilon>0$ be given. For each $s_{1} \in S_{1}$, let $\pi_{s_{1}}$ be an $\varepsilon$-optimal strategy for player I at state $s_{1} \in S_{1}$. A strategy $\pi$ for player I relying on using $\pi_{s_{1}}$ when the initial state is $s_{1}$ is an $\varepsilon$-optimal strategy for him. Thus, the result follows.

COROLLARY 4.1. If the payoff $u$ is a bounded continuous function on $H_{\infty}$, then both players have optimal strategies in the game $G$.

THEOREM 4.2. Let $G_{n}^{*}, n \in N$, be a sequence of nonstationary stochastic games satisfying (i)-(v) with payoffs $u_{n}$ such that $u_{n} \nearrow u$ as $n \rightarrow \infty$, where $u$ is the payoff of the game $G$. Then

$$
\operatorname{Val}\left(G_{n}^{*}\right) \nearrow \operatorname{Val}(G) \text { as } n \rightarrow \infty \text {. }
$$

Proof. By Theorem 4.1, each game $G_{n}^{*}$ has a value function. Moreover, in each game $G_{n}^{*}$ player II has an optimal strategy. Since $u_{n} \nearrow u$ as $n \rightarrow \infty$, so the rest of the proof proceeds along lines similar to Theorem 4.1.

Theorems 4.1 and 4.2 generalize the main results of Orkin from [7]. The methods of proofs developed here are essentially different than those used by Orkin in [7].

The conclusion of Theorem 4.2 may fail if we assume that the sequence $\left\{u_{n}\right\}$ of l.s.c. payoffs is nonincreasing and $u=\lim _{n} u_{n}$. This follows from [7, p. 215]. Here we give an example showing that the conclusion of Theorem 4.2 need not hold if $u_{n}$ and $u$ are continuous and $u_{n} \rightarrow u$ pointwise on $H_{\infty}$. This is a negative answer to an open question raised by Orkin in $[\mathbf{7}$, p. 216].

EXAMPLE 4.1. Let $S_{n}=\{n\}, X_{n}=\{0,1\}, Y_{n}=\{0\}, n \in N$. Let $d$ be a metric on $H_{\infty}$ equivalent to the product topology of $H_{\infty}$. Given any sequence $\left\{h^{n}\right\} \in H_{\infty}$ converging to $h^{0}=(0,0,0,0,0,0, \ldots)\left(h^{n} \neq h^{0}\right)$, we define a sequence $\left\{u_{n}\right\}$ of payoffs on $H_{\infty}$ as follows: $u_{n}(h)=1-d\left(h, h^{n}\right) / d\left(h^{0}, h^{n}\right)$ if $d\left(h, h^{n}\right) \leq d\left(h^{0}, h^{n}\right)$ and $u_{n}(h)=0$ otherwise. Let $u=0$. Note that $u_{n}, u$ are continuous on $H_{\infty}$ and $u_{n} \rightarrow u$ on $H_{\infty}$. Clearly, $\operatorname{Val}(G)=0$, but $\operatorname{Val}\left(G_{n}^{*}\right)=1$ for each $n \in N$.

Now, let us consider a game $G$ with independent of time state and action spaces and with a stationary law of motion (see Remark 1.1). A stationary strategy for player II in such a game is a sequence $\gamma=\left\{g_{n}\right\}$, where $g_{n}\left(h_{n}\right)=g\left(s_{n}\right)$ for each $h_{n}=\left(s_{1}, x_{1}, y_{1}, \ldots, s_{n}\right) \in H_{n}$ and some $g: S \rightarrow P(Y)$. The following example shows that player II need not have optimal stationary strategies in the game with stationary law of motion. This is a negative answer to a problem raised by Sengupta in $[\mathbf{1 0}$, Remark 4].

EXAMPLE 4.2. Let $S_{n}=S=\{0,1\}, X_{n}=\{0\}, Y_{n}=\{0,1\}, n \in N$. Let the stationary law of motion $q$ be given by $q(0,0, y, \cdot)=\delta(1)$ and $q(1,0, y, \cdot)=\delta(0)$ for each $y \in Y_{n}$. (Here $\delta(s)$ is the Dirac measure concentrated at $s \in S$.) Let 
$h^{*}=\left(s_{1}, x_{1}, y_{1}, \ldots\right) \in H_{\infty}$ be a history where $s_{2 n-1}=0, s_{2 n}=1, x_{n}=0, n \in N$,

$y_{3}=1$ and $y_{n}=0$ for $n \neq 3$. Let the payoff $u$ be defined as follows: $u(h)=0$ for $h \neq h^{*}$ and $u\left(h^{*}\right)=-1$. Note that $u$ is l.s.c. on $H_{\infty}$. Since player I has only one strategy in this game, say $\pi$, so $\operatorname{Val}(G)=\inf _{\gamma \in \Gamma} E(u, \pi, \gamma)$. It is easy to see that $\operatorname{Val}(G)(0)=-1$, but for each stationary strategy $\gamma$ of player II we have $E(u, \pi, \gamma)(0)=0$. Thus, player II has no optimal stationary strategies.

\section{REFERENCES}

1. H. A. M. Couwenbergh, Stochastic games with metric state space, Internat. J. Game Theory 9 (1980), 25-36.

2. K. Fan, Minimax theorems, Proc. Nat. Acad. Sci. U.S.A. 39 (1953), 42-47.

3. K. Hinderer, Foundations of non-stationary dymamic programming with discrete time parameter, Lecture Notes in Operations Research and Mathematical Systems 33 (M. Beckman and H. P. Künzi, eds.), Springer-Verlag, New York, 1970.

4. J. F. Mertens and A. Neyman, Stochastic games, Internat. J. Game Theory 10 (1981), 53-66.

5. J. Neveu, Mathematical foundations of the calculus of probability, Holden-Day, San Francisco, Calif., 1965.

6. A. S. Nowak, Universally measurable strategies in zero-sum stochastic games, Ann. Probab. (to appear)

7. M. Orkin, An approximation theorem for infinite games, Proc. Amer. Math. Soc. 36 (1972), 212-216.

8. T. Parthasarathy and M. Stern, Markov games - a survey, Differential Games and Control Theory. II (E. Roxin, P. Liu and R. Sternberg, eds.), Dekker, New York and Basel, 1977.

9. M. Schäl, Stochastic nonstationary two person zero sum games, Z. Angew. Math. Mech. 61 (1981), 352-353.

10. S. K. Sengupta, Lower semicontinuous stochastic games with imperfect information, Ann. Statist. 3 (1975), 554-558.

11. L. S. Shapley, Stochastic games, Proc. Nat. Acad. Sci. U.S.A. 39 (1953), 1095-1100.

Institute of MATHEMATICS, TeChNicAl UNiversity of WroclaW, 50-370 WroCŁAW, WYBRZEŻE WYSPIAŃSKIEGO 27, POLAND 